\title{
Highly sensitive plastic optical fiber with palladium sensing layer for detection of hydrogen gas
}

\begin{abstract}
This paper reports an optical sensor using plastic optical fiber for the detection of hydrogen gas. A thin layer of palladium is used as the sensing layer to enhance the performance of the optical fiber sensor. When interrogated with a broadband white light source and a spectrometer, significant changes were observed in the intensity spectrum and dynamic response of the sensor as the concentration of hydrogen in air changed. The sensor demonstrated high sensitivity towards concentrations of hydrogen between $0.5 \%$ to $2 \%$ in synthetic air with a high response and recovery time of 50 s and 80 s respectively.
\end{abstract}

Keyword: Plastic optical fiber; Optical sensor; Hydrogen; Palladium; Multimode 